\title{
Mapping of quantitative trait loci controlling heading date among rice cultivars in the northernmost region of Japan
}

\author{
Kenji Fujino* and Hiroshi Sekiguchi \\ Agricultural Research Institute, HOKUREN Federation of Agricultural Cooperatives, Higashi-5, Kita-15, Naganuma, Hokkaido 069- \\ 1317, Japan
}

\begin{abstract}
Heading date is the major factor in the regional and seasonal adaptation of rice cultivars. Although many genes controlling heading date have been identified using several mapping populations, it is not clear which previously identified genes contribute to the variation of heading date in particular regions. However, great effort is needed to identify quantitative trait loci (QTLs) among rice cultivars in rice breeding programs in a particular region because they are genetically closely related. In this study, we identified QTLs controlling heading date in $3 \mathrm{~F}_{2}$ populations among rice cultivars in Hokkaido, the northernmost region of Japan. The segregations of heading date were continuous in all populations. For QTL analysis in populations with genetically close relationships, we used SSR markers located near the known QTLs for the heading date. Three QTLs for the heading date, $q D T H 3, q D T H-6-1$, and $q D T H 6-2$, were detected on chromosomes 3 and 6 . The relationships between the three QTLs identified in this study and the known QTLs are discussed.
\end{abstract}

Key Words: rice, quantitative trait loci, heading date.

\section{Introduction}

Heading date is a major factor in regional and seasonal adaptation of rice cultivars. Due to the agronomical importance of rice, many genetic studies have been performed using several populations of varieties with diverse genetic relationships, such as inter-species crosses of cultivated rice and wild relatives (Xiao et al. 1998, Doi et al. 1998, Xiong et al. 1999, Cai and Morishima 2002, Septiningsih et al. 2003, Thomson et al. 2003), inter-subspecies crosses of indica and japonica (Li et al. 1995, Xiao et al. 1995, 1996, Lu et al. 1996, Yano et al. 1997, Lin et al. 1998, Yu et al. 2002, Mei et al. 2005, Uga et al. 2007), and relatively close genetic relationships, such as crosses among japonica varieties (Fujino and Sekiguchi 2005a, 2005b). These results revealed that many genes control heading date and this trait is under complex genetic control with interactions between the genes (Yamamoto et al. 2000, Lin et al. 2000, 2002, 2003, Gu and Foley 2007, Nonoue et al. 2008).

Although many genes controlling the heading date have been identified, it is not clear which of the previously identified genes contribute to the variation in heading date in particular regions with a sufficiently long period of favorable conditions to permit rice production. Consequently, an important aim of rice breeding programs in a particular region is to evaluate the response of a genotype under the conditions in which the cultivars will be grown and to determine

Communicated by Qian Qian

Received May 29, 2008. Accepted September 4, 2008.

*Corresponding author (e-mail: fujino-kenji@hokuren.jp) whether the gene has different effects under different environmental conditions.

Substantial efforts in rice breeding programs to improve the heading date have made rice production possible under various climatic conditions in latitudes ranging from $53^{\circ} \mathrm{N}$ to $40^{\circ} \mathrm{S}$ (Lu and Chang 1980). Because rice is a tropical shortday plant, photoperiod sensitivity (PS) plays an important role in achieving such a wide range of natural variation. For example, only extremely early-heading varieties are able to adapt to the northern limits of rice cultivation, where long natural daylength conditions prevail. This extremely early heading is expressed by extremely low PS (Tanisaka et al. 1992). The genetic basis of extremely low PS is based on two non-PS alleles at E1/qDTH7-1 and qDTH7-2 (Okumoto et al. 1996, Fujino and Sekiguchi 2005a). On the other hand, QTLs showing extremely late heading have been identified using an extremely late-heading cultivar, Nona Bokra, and a Japanese elite early-heading cultivar, Koshihikari (Uga et al. 2007). Nona Bokra exhibited markedly strong PS and Nona Bokra alleles of all 12 detected QTLs controlling heading date increased days to heading (DTH) (Uga et al. 2007).

Many mapping populations have been used to identify QTLs controlling heading date. In particular, studies using populations derived from the cross between Nipponbare and Kasalath have identified 15 QTLs for heading date, which showed small to large genetic effects (Yano et al. 1997, Lin et al. 1998, 2002, 2003, Yamamoto et al. 2000, Monna et al. 2002, reviewed by Yano et al. 2001). Among these, 9 QTLs have been mapped as single Mendelian factors (Yamamoto et al. 1998, 2000, Lin et al. 2002, 2003, Monna et al. 2002, Takeuchi et al. 2003), and some are involved in PS. The 
isolation of genes of these QTLs provides the mechanism of PS in rice comparing with that in Arabidopsis, a long-day plant (Yano et al. 2000, 2001, Takahashi et al. 2001, Kojima et al. 2002, Hayama et al. 2003, Izawa et al. 2003, Doi et al. 2004, Hayama and Coupland 2004). Based on the chromosomal location, both $q D T H 7-1$ and $q D T H 7-2$ conferring extremely early heading and 8 of 12 QTLs conferring extremely late heading may correspond to QTLs identified between Nipponbare and Kasalath (Fujino and Sekiguchi 2005a, Uga et al. 2007). The findings suggested that these QTLs identified in populations between Nipponbare and Kasalath explain the majority of a wide range of natural variation of heading date.

In this study, we identified QTLs controlling heading date among rice cultivars in Hokkaido, the northernmost region of Japan and one of the northern limits of rice cultivation. These cultivars are genetically closely related. For QTL analysis, markers around the known QTLs controlling heading date in populations between Nipponbare and Kasalath were used. In addition, the levels of polymorphism of simple sequence repeats (SSRs) markers in the population were determined.

\section{Materials and Methods}

\section{Plant materials}

Four rice cultivars from Hokkaido, Japan, were used to map QTLs controlling heading date. Hoshinoyume is a cultivar developed in 1996 in a rice breeding program in Hokkaido. Bouzu, Hokkaiwase, and Iburiwase are landracetype cultivars in Hokkaido, and were cultivated around 1900. Three $\mathrm{F}_{2}$ populations $(\mathrm{n}=86-92)$ derived from crosses of Hoshinoyume with these landrace-type cultivars were developed for mapping. $F_{2}$ populations with the parents and their $F_{1}$ plants were cultivated in a paddy field at HOKUREN Agricultural Research Institute (Naganuma, Hokkaido, Japan, $43^{\circ} 03^{\prime} \mathrm{N}$ latitude). The cultivation conditions were described in Fujino and Sekiguchi (2005a, 2005b). Sowing and transplanting were performed on April 25 and May 23, respectively, 2003. DTH of the earliest heading panicle among individuals was recorded for each plant as the number of days required from sowing to heading. Leaf samples of each plant were collected for DNA extraction.

\section{Evaluation of photoperiod sensitivity}

Hoshinoyume, Bouzu, Hokkaiwase, and Iburiwase were grown under three different environmental conditions: $16-\mathrm{h}$ long-day (16D; $16-\mathrm{h}$ light; $27^{\circ} \mathrm{C}$ for $12 \mathrm{~h}$ and $23^{\circ} \mathrm{C}$ for $12 \mathrm{~h}$ ), 14-h long-day $\left(14 \mathrm{D} ; 14-\mathrm{h}\right.$ light; $27^{\circ} \mathrm{C}$ for $12 \mathrm{~h}$ and $23^{\circ} \mathrm{C}$ for $12 \mathrm{hr}$ ), and 10 -h short-day (10D; 10 -h light; $27^{\circ} \mathrm{C}$ for $12 \mathrm{~h}$ and $23^{\circ} \mathrm{C}$ for $12 \mathrm{hr}$ ) in a controlled-growth cabinet (Nihonika, Japan). DTH required from sowing to heading was scored for 12 plants per cultivar. The mean values were calculated for each cultivar.

\section{DNA marker analysis}

It was difficult to develop polymorphic markers over the genome because the cultivars used in this study are genetically closely related. We attempted to develop polymorphic markers corresponding to the 11 known QTLs for heading date listed in Table 1, as described in Fujino and Sekiguchi (2005a). These targeted QTLs were identified using several types of progeny derived from crosses between a japonica cultivar, Nipponbare, and an indica cultivar, Kasalath (Yano et al. 1997, Lin et al. 1998, Yamamoto et al. 2000, Yano et al. 2001, Lin et al. 2002).

To amplify genomic DNA to detect SSRs, we used markers from the International Rice Microsatellite Initiative (IRMI) (McCouch et al. 2002) and previously developed SSR markers (Fujino et al. 2004). Three SSR markers near $H d 9$, GBR3003-5, were developed in this study according to the method described in Fujino et al. (2004); GBR3003 (F; AGC CAGGTATGTCATAAATGATAATAACAA, R; TGCTA TTAAACAAGCGGTTTTTTCGTTTCG), GBR3004 (F; CGCGAACGACAGCAACTGCA, R; GGACGCGGAGTT CTCCATCG), and GBR3005 (F; GGCCCAGTTGACAAA CAGGC, R; CAGAGAGCATCATGGGCCAG). In addition, one PCR primer set, Hd1, was used to detect polymorphism in the coding region of the $H d l$ gene (Fujino, unpublished data) (F; CGACGTGCAGGTGTACTCCG, R; AAT CTGTGTAAGCACTGACG). DNA was extracted from the leaves of $\mathrm{F}_{2}$ plants employing the CTAB method (Murry and Thompson 1980). PCR amplification and gel electrophoresis and detection were performed according to the method described by Fujino et al. (2004).

\section{Polymorphism of SSR markers}

To determine the availability of markers among populations with close genetic relationships, 12 rice cultivars from Japan were used: Akage, Bouzu, Bouzu No. 6, Hokkaiwase, and Iburiwase landrace types from Hokkaido; Norin No. 11, Norin No. 20, Eiko, Hoshinoyume, Honoka224, Kitaibuki, and Sasahonami breeding types from Hokkaido; and Nipponbare and Koshihikari breeding types from Honshu, the central region of Japan. Polymorphism information content (PIC) was calculated for each marker according to Nei (1973): PIC $=1-\Sigma h_{k}^{2}$, where $h_{k}$ is the frequency of the $k$ th allele.

\section{QTL analysis}

For QTL analysis of the heading date, the mean value of days to heading in $F_{2}$ populations was compared between each homozygous genotype class of parental type by ANOVA according to the procedure described in Fujino and Sekiguchi (2005a). The detection threshold for QTLs in this study was $P<0.001$. The genetic distance of the markers was calculated using MAPMAKER 3.0 employing the Kosambi function (Kosambi 1944, Lander et al. 1987).

\section{Results}

Heading behavior of cultivars

Clear varietal differences in DTH under natural field 
conditions and PS were observed among the 4 cultivars (Table 1 and Fig. 1). DTH of Hoshinoyume was 107.4 under natural field conditions, while the DTH of the other cultivars was less than Hoshinoyume (Table 1). Hoshinoyume showed the longest DTH under all daylength conditions and the greatest PS (Fig. 1). Under 10D conditions, Hokkaiwase headed the earliest. In 16D conditions, the heading delay was small in Bouzu and Iburiwase compared with under 14D conditions, while the DTH in Hoshinoyume and Hokkaiwase increased based on daylength. Due to the difference in the response to daylength, Hokkaiwase showed a larger PS than Bouzu and Iburiwase. $\mathrm{F}_{1}$ plants between Hoshinoyume and Hokkaiwase showed similar DTH to the midparent, 104.0, while both $\mathrm{F}_{1}$ plants of Hoshinoyume with Bouzu and Iburiwase headed earlier than their earlier parents (Table 1).

\section{Segregation of DTH in $F_{2}$ populations}

The DTH of $\mathrm{F}_{2}$ populations from crosses of Hoshinoyume with Bouzu, Iburiwase, or Hokkaiwase showed continuous segregation with both early and late transgressive segregants (Fig. 2). This suggested that there were several loci for heading date segregation in these crosses. The $\mathrm{F}_{2}$ population of Hoshinoyume and Hokkaiwase exhibited different segregation patterns from those of Hoshinoyume with Bouzu and Iburiwase. This included many early transgressive segregants. Together with the results of heading behavior, these cultivars might be divided into 3 genetic bases; Hoshinoyume, Hokkaiwase, and the third involves Bouzu and Iburiwase.

\section{Evaluation of marker polymorphism}

To evaluate the suitability of SSR markers to detect polymorphisms among a population with genetically close relationships, 12 and 2 cultivars from Hokkaido and Honshu, respectively, were genotyped using 51 SSR markers located around the known 11 QTLs for the heading date (Table 2). Among these, 16 SSR markers showed monomorphic results, while the others showed variations in the number of alleles and PIC values. The mean allele number was 3.28 and ranged from 2 to 11 . The mean PIC value was 0.392 and ranged from 0.133 to 0.898 . Nine SSR markers showed
Table 1. Days to heading of cultivars and their $F_{1}$ plants grown under natural field conditions

\begin{tabular}{lcc}
\hline \hline Cultivar/ $\mathrm{F}_{1}$ combination & Number of plants & $\mathrm{DTH}^{a}$ \\
\hline Hoshinoyume & 40 & $107.4 \pm 1.2$ \\
Bouzu & 40 & $103.2 \pm 1.2$ \\
Hokkaiwase & 40 & $102.9 \pm 1.6$ \\
Iburiwase & 40 & $100.9 \pm 1.4$ \\
Bouzu/Hoshinoyume & 6 & $101.0 \pm 1.1$ \\
Hoshinoyume/Hokkaiwase & 7 & $104.0 \pm 0.0$ \\
Hoshinoyume/Iburiwase & 5 & $100.8 \pm 1.1$ \\
\hline
\end{tabular}

${ }^{a}$ Plants were grown in a paddy field in 2003 .

Data represent the mean \pm SD.

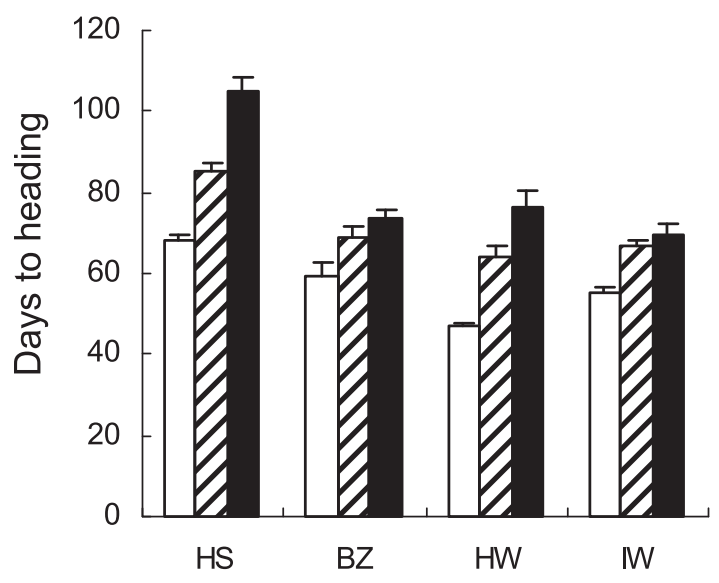

Fig. 1. DTH of rice cultivars, Hoshinoyume (HS), Bouzu (BZ), Iburiwase (IW), and Hokkaiwase (HW), under three different environmental conditions: 10D (white), 14D (hatched), and 16D (black) in a growth chamber. Data are the means and SD.

more than 6 alleles. These SSR markers with highly variable and high PIC values were distributed over the genome, indicating that they are useful to detect polymorphism in a population with genetically close relationships.

\section{QTL analysis}

The results of marker screening for the 11 known QTLs for heading date, markers with polymorphism for 10,10 ,
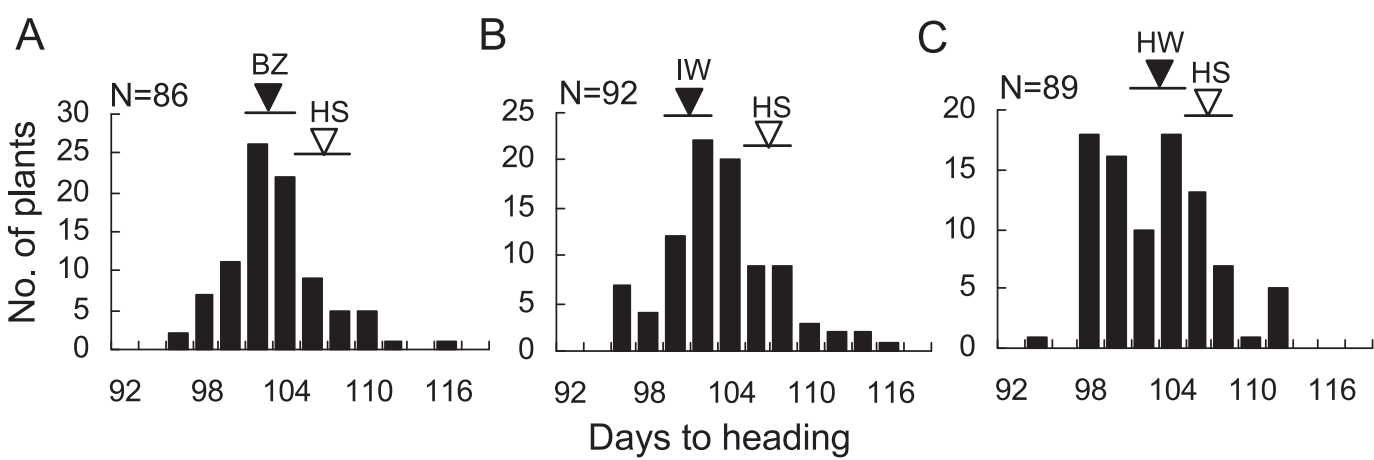

Fig. 2. Frequency distributions of DTH in different $\mathrm{F}_{2}$ populations derived from crosses of Hoshinoyume (HS) with Bouzu (BZ) (A), Iburiwase (IW) (B), and Hokkaiwase (HW) (C). Means (arrowhead) and ranges (bar) are indicated. 
Table 2. Polymorphism of markers among 14 rice cultivars in Japan

\begin{tabular}{|c|c|c|c|c|c|}
\hline Target QTL & Marker & Chr & Position $^{a}$ & $\begin{array}{l}\text { No. of } \\
\text { alleles }\end{array}$ & PIC \\
\hline \multirow[t]{3}{*}{$\overline{H d 7}$} & RM3857 & 2 & 134.5 & 2 & 0.500 \\
\hline & RM5607 & 2 & 142.5 & 1 & 0.000 \\
\hline & RM5404 & 2 & 144.7 & 2 & 0.133 \\
\hline \multirow[t]{4}{*}{$H d 9$} & GBR3002 & 3 & 2.5 & 3 & 0.604 \\
\hline & GBR3004 & 3 & 2.5 & 1 & 0.000 \\
\hline & GBR3005 & 3 & 6.3 & 2 & 0.245 \\
\hline & GBR3003 & 3 & 6.9 & 7 & 0.837 \\
\hline \multirow[t]{5}{*}{$H d 8$} & RM5393 & 3 & 38.8 & 2 & 0.337 \\
\hline & RM5442 & 3 & 38.8 & 3 & 0.520 \\
\hline & RM5444 & 3 & 38.8 & 2 & 0.245 \\
\hline & RM5639 & 3 & 39.8 & 2 & 0.245 \\
\hline & RM6417 & 3 & 39.8 & 1 & 0.000 \\
\hline \multirow[t]{2}{*}{$H d 6$} & RM5475 & 3 & 138.7 & 2 & 0.500 \\
\hline & RM5924 & 3 & 138.7 & 4 & 0.541 \\
\hline \multirow[t]{14}{*}{$H d 3$} & RM1369 & 6 & 7.4 & 4 & 0.653 \\
\hline & RM3436 & 6 & 10.4 & 4 & 0.704 \\
\hline & RM3463 & 6 & 10.4 & 4 & 0.694 \\
\hline & RM4923 & 6 & 10.4 & 7 & 0.806 \\
\hline & RM5218 & 6 & 10.4 & 11 & 0.898 \\
\hline & RM6003 & 6 & 10.4 & 1 & 0.000 \\
\hline & RM5199 & 6 & 10.4 & 7 & 0.816 \\
\hline & RM6004 & 6 & 13.5 & 1 & 0.000 \\
\hline & RM6490 & 6 & 13.5 & 1 & 0.000 \\
\hline & RM6536 & 6 & 13.5 & 1 & 0.000 \\
\hline & RM6587 & 6 & 13.5 & 1 & 0.000 \\
\hline & RM6734 & 6 & 13.8 & 2 & 0.133 \\
\hline & RM6984 & 6 & 13.8 & 3 & 0.449 \\
\hline & RM4608 & 6 & 15.8 & 8 & 0.837 \\
\hline$H d 1$ & Hd1 & 6 & 53.5 & 4 & 0.663 \\
\hline \multirow[t]{6}{*}{ Hd 4} & RM3767 & 7 & 49.4 & 2 & 0.245 \\
\hline & RM3859 & 7 & 49.4 & 3 & 0.500 \\
\hline & RM7074 & 7 & 49.4 & 3 & 0.449 \\
\hline & RM6449 & 7 & 53.4 & 1 & 0.000 \\
\hline & RM7338 & 7 & 53.4 & 1 & 0.000 \\
\hline & RM7110 & 7 & 56.2 & 3 & 0.561 \\
\hline \multirow[t]{3}{*}{$H d 2$} & RM1306 & 7 & 116.1 & 8 & 0.714 \\
\hline & RM1362 & 7 & 116.1 & 1 & 0.000 \\
\hline & RM7601 & 7 & 116.6 & 2 & 0.133 \\
\hline \multirow[t]{2}{*}{$H d 5$} & GBR8001 & 8 & 35.7 & 8 & 0.714 \\
\hline & RM5432 & 8 & 35.7 & 3 & 0.643 \\
\hline \multirow[t]{3}{*}{$H d 12$} & RM3871 & 8 & 106.1 & 4 & 0.653 \\
\hline & RM3571 & 8 & 108.2 & 1 & 0.000 \\
\hline & RM6070 & 8 & 108.2 & 1 & 0.000 \\
\hline \multirow{7}{*}{ Hd14 } & RM5304 & 10 & 41.9 & 4 & 0.582 \\
\hline & RM6962 & 10 & 42.7 & 2 & 0.459 \\
\hline & RM6924 & 10 & 43.7 & 8 & 0.806 \\
\hline & RM5392 & 10 & 44.9 & 1 & 0.000 \\
\hline & RM5620 & 10 & 44.9 & 1 & 0.000 \\
\hline & RM5756 & 10 & 48.8 & 1 & 0.000 \\
\hline & RM6704 & 10 & 48.8 & 3 & 0.622 \\
\hline \multirow[t]{2}{*}{$H d 13$} & RM5653 & 12 & 47.0 & 6 & 0.776 \\
\hline & & & Average & 3.28 & 0.392 \\
\hline
\end{tabular}

${ }^{a}$ Position of the markers on the high-density linkage map (Harushima et al. 1998). and 11 QTLs, were identified for the combination of Hoshinoyume with Bouzu, Hokkaiwase, and Iburiwase, respectively (Table 3). QTL analysis for heading date was performed by ANOVA using genotypes of these markers and DTH (Table 4). No QTLs were detected in the $\mathrm{F}_{2}$ population from the cross between Hoshinoyume and Hokkaiwase, while 2 and 3 QTLs were detected from crosses of Hoshinoyume with Bouzu and Iburiwase, respectively.

Two QTLs in the $\mathrm{F}_{2}$ population from the cross between Hoshinoyume and Bouzu were located on the short arm of chromosome 6; qDTH6-1 detected with marker RM5199 and $q D T H 6-2$ with marker Hd1. For both loci, Bouzu alleles increased DTH; 5.0 days for $q D T H 6-1$ and 4.8 days for qDTH6-2. Three QTLs in the $\mathrm{F}_{2}$ population from the cross between Hoshinoyume and Iburiwase were located on the short arm of chromosomes 3 and 6; qDTH3 detected with marker GBR3003, qDTH6-1 with marker RM4608, and qDTH6-2 with marker Hd1. Iburiwase alleles of qDTH6-1 and $q D T H 6-2$ increased DTH, by 4.0 and 7.3 days, respectively, while that of $q D T H 3$ decreased DTH by 6.6 days. The genetic distances between markers RM5199/RM4608 for qDTH6- 1 and $\mathrm{Hd} 1$ for $q D T H 6-2$ were 59.1 and $30.7 \mathrm{cM}^{\text {in }} \mathrm{F}_{2}$ populations from crosses of Hoshinoyume with Bouzu and Iburiwase, respectively. This indicated that these 2 QTLs were independent of each other.

\section{Discussion}

Identification of QTLs controlling heading date

The manipulation of heading date is an important objective in rice breeding programs for the adaptation of cultivars to a particular region in which they will grow. In this study, to understand the genetic basis of DTH among rice cultivars from Hokkaido, which showed genetically close relationships, we performed QTL mapping using 3 different populations from crosses between cultivars from recent rice breeding programs and landrace types. Although the variation of DTH under natural field conditions was small (Table 1), the

Table 3. Number of SSR markers surveyed for polymorphisms in crosses of Hoshinoyume with 3 cultivars

\begin{tabular}{|c|c|c|c|c|c|}
\hline \multirow{2}{*}{$\begin{array}{l}\text { Target } \\
\text { QTL }\end{array}$} & \multirow{2}{*}{ Chr } & \multirow{2}{*}{$\begin{array}{l}\text { No. of markers } \\
\text { tested }\end{array}$} & \multicolumn{3}{|c|}{ No. of polymorphic markers } \\
\hline & & & Bouzu & Hokkaiwase & Iburiwase \\
\hline$\overline{H d 7}$ & 2 & 3 & 1 & 2 & 1 \\
\hline$H d 9$ & 3 & 4 & 2 & 2 & 1 \\
\hline$H d 8$ & 3 & 5 & 2 & 2 & 2 \\
\hline$H d 6$ & 3 & 2 & 2 & 2 & 1 \\
\hline$H d 3$ & 6 & 14 & 8 & 7 & 8 \\
\hline$H d 1$ & 6 & 1 & 1 & 1 & 1 \\
\hline$H d 4$ & 7 & 6 & 0 & 0 & 1 \\
\hline$H d 2$ & 7 & 3 & 1 & 2 & 0 \\
\hline$H d 5$ & 8 & 2 & 2 & 2 & 2 \\
\hline$H d 12$ & 8 & 3 & 0 & 0 & 1 \\
\hline$H d 14$ & 10 & 7 & 4 & 4 & 3 \\
\hline$H d 13$ & 12 & 1 & 1 & 1 & 1 \\
\hline
\end{tabular}


Table 4. QTLs of heading date in $\mathrm{F}_{2}$ populations as determined by ANOVA

\begin{tabular}{|c|c|c|c|c|c|c|c|c|c|c|c|c|}
\hline \multirow[b]{2}{*}{ Combination (A/B) } & \multirow[b]{2}{*}{$\begin{array}{c}\text { Target } \\
\text { QTL }\end{array}$} & \multirow[b]{2}{*}{ Marker } & \multirow[b]{2}{*}{ Chr } & \multirow[b]{2}{*}{ Position $^{a}$} & \multirow{2}{*}{$\begin{array}{l}\text { No. of } \\
\text { plants } \\
\text { tested }\end{array}$} & \multicolumn{6}{|c|}{ Mean of DTH } & \multirow[b]{2}{*}{ Probability } \\
\hline & & & & & & $\begin{array}{l}\mathrm{A}^{b} \\
\mathrm{n}\end{array}$ & & $\begin{array}{l}\mathrm{B}^{b} \\
\mathrm{n}\end{array}$ & & $\begin{array}{l}\mathrm{H}^{b} \\
\mathrm{n}\end{array}$ & & \\
\hline \multirow[t]{3}{*}{ Bouzu/Hoshinoyume } & $H d 9$ & GBR3002 & 3 & 2.5 & 73 & 23 & 102.3 & 17 & 104.1 & 33 & 103.8 & 0.1042 \\
\hline & $H d 3$ & RM5199 & 6 & 10.4 & 86 & 18 & 105.7 & 18 & 100.7 & 50 & 103.3 & $0.0001 *$ \\
\hline & $H d 1$ & Hd1 & 6 & 50.0 & 89 & 15 & 106.7 & 31 & 101.9 & 43 & 103.0 & $0.0000^{*}$ \\
\hline \multirow[t]{3}{*}{ Hoshinoyume/Hokkaiwase } & $H d 9$ & GBR3002 & 3 & 2.5 & 87 & 24 & 104.8 & 20 & 101.9 & 43 & 102.3 & 0.0168 \\
\hline & $H d 3$ & RM4608 & 6 & 15.8 & 80 & 17 & 102.7 & 22 & 103.6 & 41 & 102.6 & 0.4889 \\
\hline & $H d 1$ & Hd1 & 6 & 50.0 & 89 & 24 & 101.3 & 15 & 104.8 & 50 & 103.0 & 0.0122 \\
\hline \multirow[t]{3}{*}{ Hoshinoyume/Iburiwase } & $H d 9$ & GBR3003 & 3 & 6.9 & 91 & 25 & 106.7 & 20 & 100.1 & 46 & 103.1 & $0.0000^{*}$ \\
\hline & $H d 3$ & RM4608 & 6 & 15.8 & 90 & 19 & 100.9 & 18 & 104.9 & 53 & 103.9 & $0.0006^{*}$ \\
\hline & $H d l$ & Hd1 & 6 & 50.0 & 91 & 27 & 100.4 & 22 & 107.7 & 42 & 102.8 & $0.0000^{*}$ \\
\hline
\end{tabular}

Results in the QTLs regions identified in this study.

* The detection threshold for QTLs was $P<0.001$ between the mean of DTH of A and B types.

${ }^{a}$ Position of the markers on the high-density linkage map (Harushima et al. 1998).

${ }^{b} \mathrm{~A}, \mathrm{~B}$, and $\mathrm{H}$ indicate homozygous $\mathrm{A}$ and $\mathrm{B}$ parental types and heterozygous alleles, respectively. A and B indicate female and male parents in each population.

genotype of heading date and heading behavior were different among these cultivars (Fig. 1 and Fig. 2). As a result, we detected 3 QTLs for heading date, qDTH3, qDTH6-1, and $q D T H 6-2$, on chromosomes 3 and 6 (Table 4).

\section{Relationships between our QTLs and known QTLs}

Comparisons of chromosomal locations suggested that $q D T H 3, q D T H 6-1$, and $q D T H 6-2$ identified in this study may be the same as $H d 9$ (Lin et al. 2002), Hd3 (Yano et al. 1997), and Hdl (Yano et al. 1997), respectively. Hdl (Sel) is a major PS gene (Yokoo and Kikuchi 1977, Yano et al. 1997, Lin et al. 2000), an ortholog of CONSTANS in Arabidopsis (Yano et al. 2000), and plays an important role in PS (Yano et al. 2000, Izawa et al. 2003, Hayama and Coupland 2004). The $H d 3$ locus contains two loci, $H d 3 a$ and $H d 3 b$, which are involved in PS (Monna et al. 2002). Hd3a is an ortholog of FT in Arabidopsis (Kojima et al. 2002). On the other hand, the function of $H d 9$ has not been determined clearly (Lin et al. 2002). In addition, many genes corresponding to these chromosomal regions have been identified using populations with genetically wide relationships (Yano et al. 1997, Lin et al. 2002, Monna et al. 2002, summarized in Gramene; http://www.gramene.org/). It was difficult to determine whether the QTLs identified in this study were the same as loci reported using other populations or were unidentified loci linked to these reported loci based on the comparison of chromosomal location. Allelic tests or identification of their precise chromosomal locations will be needed.

The QTLs corresponding to the 3 QTLs identified in this study play important roles in PS in rice and show large phenotypic effects; however, the QTLs identified in this study showed relatively small effects. For example, qDTH6-2 identified in this study showed relatively small effects of 5 and 7 days' difference in populations derived from crosses of Hoshinoyume with Bouzu and Iburiwase, respectively (Table 4). Why could these genes with large effects not ex- press their functions in cultivars from Hokkaido? Recently, Nonoue et al. (2008) demonstrated that epistatic interactions contribute to extremely early heading. Hoshinoyume carries the same functional allele of the Hdl gene as Ginbouzu, a late-heading cultivar (Yano et al. 2000, Nonoue et al. 2008). The deduced phenotypic effect of $H d l$ was caused by the epistatic interaction between Hdl and QTL (chr7) (Nonoue et al. 2008). Under the genotype of a non-PS allele at QTL (chr7), Hdl could not express strong PS (Nonoue et al. 2008). Based on the chromosomal location, QTL (chr7) may correspond to E1, Hd4, and qHDT7-1 (Yano et al. 1997, Ichitani et al. 1998, Fujino and Sekiguchi 2005a). To express extremely early heading, a non-PS allele at E1/qDTH7-1 is essential (Okumoto et al. 1996, Fujino and Sekiguchi 2005a); therefore, the results of this study revealed that $q$ DTH6-2 plays an important role in the variation of heading date among cultivars with extremely low PS.

Strategy of QTL detection among populations with genetically close relationships

The varietal difference of DTH between Hoshinoyume and Iburiwase, 6.3 days, was almost explained by the additive effect of the 3 QTLs: +6.6 days by $q D T H 3,-4.0$ days by qDTH6-1, and -7.3 days by qDTH6-2 for Hoshinoyume alleles. Although the difference in DTH between Hoshinoyume and Bouzu was 4.2 days, Hoshinoyume alleles of both QTLs decreased DTH, -5.0 days by qDTH6-1 and -4.8 days by $q D T H 6-2$. Unidentified QTLs controlling the heading date for the difference between Hoshinoyume and Bouzu remained. One possibility is that unidentified QTLs may be located at sites other than the QTLs tested in this study. The other possibility is epistatic interactions between the genes underlying them. The population size in this study, $<100$ plants, was not sufficient for statistical analysis of epistatic interactions in each genotype class. It is necessary to use near-isogenic lines for each QTL to identify epistatic 
interactions between them.

In contrast to the above cases, no QTL for the heading date was detected in the $\mathrm{F}_{2}$ population derived from the cross between Hoshinoyume and Hokkaiwase (Table 4). The same reasons as mentioned above were considered for the failure to detect further QTLs. Hokkaiwase showed unique PS, DTH of the $\mathrm{F}_{1}$ plants in the cross with Hoshinoyume, and the unique distribution of DTH in the $\mathrm{F}_{2}$ population (Fig. 1 and Fig. 2, Table 1). This suggested that other than the 3 QTLs identified in this study, further QTLs play major roles in the difference in DTH between Hoshinoyume and Hokkaiwase. QTL analysis of the whole genome may discover new loci/ alleles controlling the heading date.

\section{Conclusion}

Due to intensive breeding efforts, the genetic diversity of modern rice cultivars has been reduced (Dilday 1990, Cuevas-Perez et al. 1992). Although transgressive segregations of DTH were observed in $\mathrm{F}_{2}$ populations derived from crosses between cultivars in recent rice breeding programs and in landrace types in this study (Fig. 2), a single PS gene, $S e(t)$, confers heading date variations among cultivars in recent rice breeding programs in Hokkaido (Fujino 2003), which may correspond to Hd5 (Nonoue et al. 2008). These results indicated that genetic differentiation at $S e(t), q D T H 6-1$, and $q D T H 6-2$ may play important roles in controlling DTH in rice breeding programs in Hokkaido.

Combining information about known QTLs with the extensive screening of SSR markers makes it possible to identify QTLs controlling the heading date conferring variations among cultivars adapted to a particular region. Understanding the genetic basis in breeding materials will be useful to manipulate the heading date in rice breeding programs. Because the interaction of many genes is involved in agronomically important traits, effective utilization of a new germplasm in marker-assisted selection (MAS) strategies in rice-breeding programs is needed to understand the genotypes of cultivars.

\section{Literature Cited}

Cai,H.W. and H.Morishima (2002) QTL clusters reflect character associations in wild and cultivated rice. Theor. Appl. Genet. 104: $1217-1228$.

Cuevas-Perez,F.E., E.P. Guimaraes, L.E. Berrio and D.I.Gonzalez (1992) Genetic base of irrigated rice in Latin America and the Caribbean, 1971 to 1989. Crop Sci. 32: 1054-1059.

Dilday, R.H. (1990) Contribution of ancestral lines in the development of new cultivars of rice. Crop Sci. 30: 905-911.

Doi,K., A.Yoshimura and N.Iwata (1998) RFLP mapping and QTL analysis of heading date and pollen sterility using backcross population between Oryza sativa L. and Oryza glaberrima Steud. Breed. Sci. 48: 395-399.

Doi, K., T. Izawa，T.Fuse，U.Yamanouchi，T.Kubo，Z.Shimatani, M.Yano and A. Yoshimura (2004) Ehdl, a B-type response regulator in rice, confers short-day promotion of flowering and controls $F T$-like gene expression independently of $H d l$. Genes Dev. 18 :
926-936.

Fujino,K. (2003) Photoperiod sensitivity gene controlling heading date in rice cultivars in the northernmost region of Japan. Euphytica 131: 97-103.

Fujino,K., H.Sekiguchi, T.Sato, H.Kiuchi, Y.Nonoue, Y.Takeuchi, T.Ando, S.Y.Lin and M.Yano (2004) Mapping of quantitative trait loci controlling low-temperature germinability in rice (Oryza sativa L.). Theor. Appl. Genet. 108: 794-799.

Fujino,K. and H.Sekiguchi (2005a) Mapping of QTLs conferring extremely early heading in rice (Oryza sativa L.). Theor. Appl. Genet. 111: 393-398.

Fujino,K. and H.Sekiguchi (2005b) Identification of QTLs conferring genetic variation for heading date among rice varieties at the northern-limit of rice cultivation. Breed. Sci. 55: 141-146.

Gu,X.Y. and M.E.Foley (2007) Epistatic interactions of three loci regulate flowering time under short and long daylengths in a backcross population of rice. Theor. Appl. Genet. 114: 745-754.

Harushima, Y., M.Yano, A.Shomura, M.Sato, T.Shimano, Y.Kuboki, T. Yamamoto, S.Y.Lin, B.A. Antonio, A. Parco, H. Kajiya, N.Huang, K.Yamamoto, Y.Nagamura, N.Kurata, G.S.Khush and T.Sasaki (1998) A high-density rice genetic linkage map with 2275 markers using a single $\mathrm{F}_{2}$ population. Genetics 148: 479-494.

Hayama, R., S.Yokoi, S.Tamaki, M.Yano and K. Shimamoto (2003) Adaptation of photoperiodic control pathways produces short-day flowering in rice. Nature 422: 719-722.

Hayama, R. and G.Coupland (2004) The molecular basis of diversity in the photoperiodic flowering responses of Arabidopsis and rice. Plant Physiol. 135: 677-684.

Ichitani,K., Y.Okumoto and T.Tanisaka (1998) Genetic analysis of the rice cultivar Kasalath with special reference to two photoperiod sensitivity loci, $E 1$ and $\mathrm{Se}-1$. Breed. Sci. 48: 51-57.

Izawa,T., Y.Takahashi and M. Yano (2003) Comparative biology comes into bloom: genomic and genetic comparison of flowering pathways in rice and Arabidopsis. Curr. Opin. Plant Biol. 6: 113120.

Kojima,S., Y.Takahashi, Y.Kobayashi, L.Monna, T.Sasaki, T.Araki and M. Yano (2002) Hd3a, a rice ortholog of the Arabidopsis FT gene, promotes transition to flowering downstream of $H d l$ under short-day conditions. Plant Cell Physiol. 10: 1096-1105.

Kosambi,D.D. (1944) The estimation of map distances from recombination value. Ann. Eugenet. 12: 172-175.

Lander,E.S., P.Green, J.Abrahamson, A. Barlow, M.J.Daly, S.E. Lincoln and L.Newburg (1987) MAPMAKER: an interactive computer package for constructing primary genetic linkage maps of experimental and natural populations. Genomics 1: 174-181.

Li,Z.K., S.R.M.Pinson, J.W.Stansel and W.D.Park (1995) Identification of quantitative trait loci (QTLs) for heading date and plant heigth in cultivated rice (Oryza Sativa L.). Theor. Appl. Genet. 91: 374-381.

Lin,H.X., T.Yamamoto, T. Sasaki and M.Yano (2000) Characterization and detection of epistatic interactions of three QTLs, Hdl, $H d 2$ and $H d 3$, controlling heading date in rice using nearly isogenic lines. Theor. Appl. Genet. 101: 1021-1028.

Lin,H.X., M.Ashikari, U.Yamanouchi, T.Sasaki and M.Yano (2002) Identification and characterization of a quantitative trait locus, $H d 9$, controlling heading date in rice. Breed. Sci. 52: 35-41.

Lin,H.X., Z.W.Liang, T.Sasaki and M.Yano (2003) Fine mapping and characterization of quantitative trait loci $H d 4$ and $H d 5$ controlling heading date in rice. Breed. Sci. 53: 51-59.

Lin,S.Y., T.Sasaki and M.Yano (1998) Mapping quantitative trait loci controlling seed dormancy and heading date in rice, Oryza sativa 
L., using backcross inbred lines. Theor. Appl. Genet. 96: 9971003.

Lu,C., L.Shen, Z.Tan, Y.Xu, P.He, Y.Chen and L.Zhu (1996) Comparative mapping of QTLs for agronomic traits of rice across environments using a double-haploid population. Theor. Appl. Genet. 93: 1211-1217.

Lu,J.J. and T.T.Chang (1980) Rice in its temporal and spatial perspectives. In: Luh,B.S. (ed.) Rice: Production and utilization. AVI Publishing Co., Inc., Westport, CT, pp. 1-74.

McCouch,S.R., L.Teytelman, Y.Xu, K.B.Lobos, K.Clare, M.Walton, B.Fu, R.Maghirang, Z.Li, Y.Xing et al. (2002) Development and mapping of 2240 new SSR markers for rice (Oryza sativa L.). DNA Res. 9: 199-207.

Mei,H.W., Z.K.Li, Q.Y.Shu, L.B.Guo, Y.P.Wang, X.Q.Yu, C.S.Ying and L.J.Luo (2005) Gene actions of QTLs affecting several agronomic traits resolved in a recombinant inbred rice population and two backcross populations. Theor. Appl. Genet. 110: 649-659.

Monna,L., H.X.Lin, S.Kojima, T.Sasaki and M.Yano (2002) Genetic dissection of a genomic region for a quantitative trait locus, $H d 3$, into two loci, $H d 3 a$ and $H d 3 b$, controlling heading date in rice. Theor. Appl. Genet. 104: 772-778.

Murray,M.G. and W.F.Thompson (1980) Rapid isolation of high molecular weight plant DNA. Nucl. Acids. Res. 8: 4321-4325.

Nei,M. (1973) Analysis of gene diversity in subdivided populations. Proc. Natl. Acad. Sci. USA 70: 3321-3323.

Nonoue, Y., K.Fujino, Y.Hirayama, U.Yamanouchi, S.Y.Lin and M.Yano (2008) Detection of quantitative trait loci controlling extremely early heading in rice. Theor. Appl. Genet. 116: 715-722.

Okumoto, Y., K.Ichitani, H.Inoue and T.Tanisaka (1996) Photoperiod insensitivity gene essential to the varieties grown in the northern limit region of paddy rice (Oryza sativa L.) cultivation. Euphytica 92: 63-66.

Septiningsih,E.M., J.Prasetiyono, E.Lubis, T.H.Tai, T.Tjubaryat, S.Moeljopawiro and S.R.McCouch (2003) Identification of quantitative trait loci for yield and yield components in an advanced backcross population derived from the Oryza sativa variety IR64 and the wild relative O. rufipogon. Theor. Appl. Genet. 107: 14191432.

Takahashi,Y., A.Shomura, T.Sasaki and M.Yano (2001) Hd6, a rice quantitative trait locus involved in photoperiod sensitivity, encodes the $\alpha$ subunit of protein kinase $C K 2$. Proc. Natl. Acad. Sci. USA 98: 7922-7927.

Takeuchi,Y., S.Y.Lin, T.Sasaki and M.Yano (2003) Fine linkage mapping enables dissection of closely linked quantitative trait loci for seed dormancy and heading in rice. Theor. Appl. Genet. 107: 1174-1180.

Tanisaka,T., H.Inoue, S.Uozu and H.Yamagata (1992) Basic vegitative growth and photoperiod sensitivity of heading-time mutants induced in rice. Jpn J. Breed. 42: 657-668.

Thomson, M.J., T.H. Tai, A.M. McClung, X.H. Lai, M.E.Hinga,
K.B.Lobos, Y.Xu, C.P.Martinez and S.R.McCouch (2003) Mapping quantitative trait loci for yield, yield components and morphological traits in an advanced backcross population between Oryza rufipogon and the Oryza sativa cultivar Jefferson. Theor. Appl. Genet. 107: 479-493.

Uga, Y., Y.Nonoue, Z.W.Liang, H.X.Lin, S.Yamamoto, U.Yamanouchi and M.Yano (2007) Accumulation of additive effects generates a strong photoperiod sensitivity in the extremely late-heading rice cultivar 'Nona Bokra'. Theor. Appl. Genet.114: 1457-1466.

Xiao,J., J.Li, L.Yuan and S.D.Tanksley (1995) Dominance is the major genetic basis of heterosis in rice as revealed by QTL analysis using molecular markers. Genetics 140: 745-754.

Xiao,J., J.Li, L.Yuan and S.D.Tanksley (1996) Identification of QTLs affecting traits of agronomic importance in a recombinant inbred population derived from a subspecific rice cross. Theor. Appl. Genet. 92: 230-244.

Xiao,J., J.Li, S.Grandillo, S.N.Ahn, L.Yuan, S.D. Tanksley and S.R.McCouch (1998) Identification of trait-improving quantitative trait loci alleles from a wild rice relative, Oryza rufipogon. Genetics 150: 899-909.

Xiong,L.Z., K.D.Liu, X.K.Dai, C.G.Xu and Q.Zhang (1999) Identification of genetic factors controlling domestication-related traits of rice using an $\mathrm{F}_{2}$ population of a cross between Oryza sativa and O. rufipogon. Theor. Appl. Genet. 98: 243-251.

Yamamoto,T., Y.Kuboki, S.Y.Lin, T.Sasaki and M.Yano (1998) Fine mapping of quantitative trait loci $H d-1, H d-2$ and $H d-3$, controlling heading date of rice, as single Mendelian factors. Theor. Appl. Genet. 97: 37-44.

Yamamoto,T., H.X.Lin, T.Sasaki and M.Yano (2000) Identification of heading date quantitative trait locus $H d 6$ and characterization of its epistatic interactions with $H d 2$ in rice. Genetics 154: 885-891.

Yano, M., Y.Harushima, Y.Nagamura, N.Kurata, Y.Minobe and T.Sasaki (1997) Identification of quantitative trait loci controlling heading date in rice using a high-density linkage map. Theor. Appl. Genet. 95: 1025-1032.

Yano, M., Y. Katayose, M. Ashikari, U. Yamanouchi, L. Monna, T.Fuse, T.Baba, K.Yamamoto, Y.Umehara, Y.Naganura and T.Sasaki (2000) $H d l$, a major photoperiod sensitivity quantitative trait locus in rice, is closely related to the Arabidopsis flowering time gene CONSTANS. Plant Cell 12: 2473-2484.

Yano,M., S.Kojima, Y.Takahashi, H.X.Lin and T. Sasaki (2001) Genetic control of flowering time in rice, a short-day plant. Plant Physiol. 127: 1425-1429.

Yokoo,M. and F.Kikuchi (1977) Multiple allelism of the locus controlling heading time of rice, detected using close linkage with blast-resistance. Japan J. Breed. 21: 123-130.

Yu,S.B., J.X.Li, C.G.Xu, Y.F.Tan, X.H.Li and Q.Zhang (2002) Identification of quantitative trait loci and epistatic interactions for plant height and heading date in rice. Theor. Appl. Genet. 104: 619-625. 